N1682

Germanistische Abhandlungen

Politische Typologie und dRAmatisches Exemplum 


\section{Politische Typologie und \\ dramatisches Exemplum}

Studien zum historisch-ästhetischen Horizont

des barocken Trauerspiels am Beispiel von Andreas Gryphius'

Carolus Stuardus und Papinianus 


\section{GERMANISTISCHE ABHANDLUNGEN 55}

Die vorliegende Arbeit ist die überarbeitete Fassung der im Sommer 1979 von der Neuphilologischen Fakultät der Universität Heidelberg angenommene Dissertation "Politische Typologie und dramatisches Exemplum. Studien zum historisch-ästhetischen Horizont des barocken Trauerspiels am Beispiel von Andreas Gryphius' Carolus Stuardus und Papinianus."

1. Gutachter: Prof. Dr. Peter Michelsen

2. Gutachter: Prof. Dr. Arthur Henkel

Tag der mündlichen Prüfung: 4. Juli 1979

CIP-Kurztitelaufnahme der Deutschen Bibliothek

Habersetzer, Karl-Heinz:

Politische Typologie und dramatisches Exemplum:

Studien zum histor.-ästhet. Horizont d. barocken Trauerspiels am Beispiel von Andreas Gryphius' "Carolus Stuardus « und "Papinianus« / Karl-Heinz Habersetzer. Stuttgart: Metzler, 1985.

(Germanistische Abhandlungen; 55)

ISBN 978-3-476-00571-7

ISBN 978-3-476-03206-5 (eBook)

DOI 10.1007/978-3-476-03206-5

NE: Gryphius, Andreas: Papinianus; GT

(c) 1985 Springer-Verlag GmbH Deutschland

Ursprünglich erschienen bei J. B. Metzlersche Verlagsbuchhandlung und Carl Ernst Poeschel Verlag GmbH in Stuttgart 1985 
FÜR ULRIKE 
Ich wurde am 11. September 1940 als Sohn des Karl Habersetzer und seiner Ehefrau Anni in Heidelberg geboren.

Hier besuchte ich von 1947 bis 1955 die Volksschule und daran anschließend bis 1957 die Höhere Handelsschule. Von 1957 bis 1960 wurde ich in verschiedenen Firmen und Banken zum Industrie- und Bankkaufmann ausgebildet und legte die Kaufmannsgehilfenprüfung ab.

Es folgte das humanistische Abitur am Aufbaugymnasium der Heimschule Lender in Sasbach/Baden. Von 1965 bis 1971 studierte ich an der Universität Heidelberg Germanistik, Geschichte und Philosophie. Meine Lehrer waren die Professoren: Peter Claasen, Werner Conze, Hans-Georg Gadamer, Christian Habicht, Arthur Henkel, Eberhard Lämmert, Peter Michelsen, Peter von Polenz, Roswitha Wisniewski. Im Sommersemester 1971 legte ich das erste Staatsexamen ab.

Von 1971 bis 1973 war ich Angestellter der Deutschen Forschungsgemeinschaft mit Sitz in der Herzog August Bibliothek in Wolfenbüttel und wechselte von hier 1973 auf die Stelle eines wissenschaftlichen Assistenten am Lehrstuhl für Deutsche Sprache und Literatur der Technischen Universität Braunschweig. 1979/80 war ich Gastprofessor an der University of Cincinnati (Ohio, USA), nachdem ich 1979 an der Universität Heidelberg das Doktorexamen bestanden hatte. 1982 legte ich das zweite Staatsexamen für das Lehramt an Gymnasien ab und befinde mich seit dieser Zeit im Schuldienst des Landes Baden-Württemberg. 
Die vorliegende Untersuchung ist die überarbeitete Fassung meiner im Sommer 1979 von der Neuphilologischen Fakultät der Universität Heidelberg angenommenen Dissertation über die Trauerspiele des Andreas Gryphius. Bei der Überarbeitung wurde soweit wie möglich auf die zu wesentlichen Themen der Darstellung erschienene Literatur verwiesen; die Thesen der Arbeit blieben davon unberührt.

Mein Lehrer, Professor Peter Michelsen, hat die Arbeit angeregt und ihren Fortgang mit kritischem Interesse verfolgt; dafür und für viele ebenso anregende wie offene Gespräche habe ich ihm herzlich zu danken.

Herrn Professor Arthur Henkel, der mich in die Literatur des Barock einführte, verdanke ich wichtige Korrigenda zu einzelnen Kapiteln der Arbeit.

Es war ein besonderer Glücksfall, daß ich über Jahre hinaus aus den Quellen der Herzog August Bibliothek in Wolfenbüttel schöpfen konnte und durch den dort regen wissenschaftlichen Diskurs vielfältige Anregungen erfahren durfte. Herrn Professor Paul Raabe, der mir zuerst als seinem Mitarbeiter, dann als schlichtem Benutzer den Zugang zu den Schätzen der Bibliothek verschaffte, habe ich für die so genossene Barocke Bücherlust zu danken.

Viele Freunde und Kollegen haben mich beim Entstehen und Fortgang der Arbeit mit ihrem Interesse, hilfreichen Gesprächen und wichtigen Hinweisen entscheidend gefördert. Ihnen allen bin ich zu großem Dank verpflichtet: Martin Bircher, Emilio Bonfatti, Gerhard Dünnhaupt, Gotthardt Frühsorge, Franz Heiduk, Sibylle Penkert, Christoph Perels, Eberhard Rohse, Elida Maria Szarota und Marian Szyrocki. Vom Metzler-Verlag habe ich Herrn Dr. Bernd Lutz für vielseitige Unterstützung zu danken. Der VG Wort in München gilt mein Dank für einen namhaften Druckkostenzuschuß.

Ohne die stetige Hilfe und Rücksicht meiner lieben Frau wäre die Arbeit wohl kaum fertiggestellt worden. Ihr ist sie gewidmet. 


\section{INHALTSVERZEICHNIS}

VORWORT ...................... VII

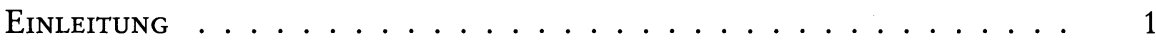

Politische Typologie und dramatisches Exemplum . . . . . . . . . . . 1

I. Politische Typologie . . . . . . . . . . . . . . . . . 15

1. Carolus Stuardus. Trauer-Spil . . . . . . . . . . . . . . . . . . 15

2. Shakespeare:RichardII. ............... 43

II. Politische Typologie als Handlungsintention . . . . . . . . . . 46 Zur Widmung des Carolus Stuardus an den Großen Kurfürsten von Brandenburg .................. 46

III. Dramatisches Exemplum: Papinianus . . . . . . . . . . . . 60

1. Exemplum als Moralphilosophie: Magnanimitas . . . . . . . . . 60

2. Themis und Gewissen: Mythologisches Exemplum als dramatische Theodizee . . . . . . . . . . . . . 71

3. Staatsräson: Necessitas und Fortuna . . . . . . . . . . . . . . . . . . 82

4. Widerstandsrecht:Jean Bodin und Hugo Grotius . . . . . . . . . . . 87

IV. Dramatisches Exemplum und Rezeptionsgeschichte . . . . . . . . . 95

Andreas Gryphius: Papinianus. Trauer-Spil.1659 . . . . . . . . . . . . . 95

Franz Neumayr, S. J.: Papinianus. Tragoedia. 1733 . . . . . . . . . . . . 95

ANHANG . . . . . . . . . . . . . . . . . . 129

ANDREAs Gryphius' HochzeItSgedicht für Gabriel Luther . . . . . 130

Franz Neumayr, S. J.: Papinianus. Tragoedia.-Textedition. . . . . . . . 134 
$\mathrm{X}$

VERZEICHNIS DER AbKüRZUNGEN . . . . . . . . . . . . . . . . . . . 165

ANMERKUngEN . . . . . . . . . . . . . . . . . . . 166

LITERATURVERZEICHNIS . . . . . . . . . . . . . . . . . . . . 217

ReGISTER . . . . . . . . . . . . . . . . . . . . . . 242 